\title{
Leak-free Group Signatures with Immediate Revocation
}

\author{
Xuhua Ding* \\ School of Information Systems \\ Singapore Management University \\ xhding@smu.edu.sg
}

\author{
Gene Tsudik \\ Department of Computer Science \\ University of California, Irvine \\ gts@ics.uci.edu
}

\author{
Shouhuai $\mathrm{Xu}^{\dagger}$ \\ Department of Computer Science \\ University of Texas at San Antonio \\ shxu@cs.utsa.edu
}

\begin{abstract}
Group signatures are an interesting and appealing cryptographic construct with many promising potential applications. This work is motivated by attractive features of group signatures, particularly, their potential to serve as foundation for anonymous credential systems. We re-examine the entire notion of group signatures from a systems perspective and identify two new security requirements: leak-freedom and immediate-revocation, which are crucial for a large class of applications. We then present a new group signature scheme that achieves all identified properties. Our scheme is based on the so-called systems architecture approach. It is more efficient than the state-of-the-art and facilitates easy implementation. Moreover, it reflects the wellknown separation-of-duty principle. Another benefit of our scheme is the obviated reliance on underlying anonymous communication channels, which are necessary in previous schemes.
\end{abstract}

\section{Introduction}

The concept of group signatures was introduced by Chaum and van Heyst [18] in 1991. The chief motivation was to facilitate anonymous group-oriented applications. Given a valid group signature, any verifier can determine the signer's group membership, while only a designated entity (called a group manager) can identify the actual signer. In recent years, many research efforts sought efficient constructs and precise definitions of group signatures. Early schemes (e.g., [19]) suffered from lin-

* Work done at the University of California, Irvine.

$\dagger$ Work done, in part, at the University of California, Irvine. ear complexities of either (or both) group public key size or signature size with respect to the number of group members. These drawbacks were first addressed by Camenisch and Stadler [13]. Follow-on results [12,1] gradually improve on efficiency. Despite these advances, membership revocation remained a difficult obstacle $[7,33,2]$. Significant progress was made by Camenisch and Lysianskaya [9] who utilized a novel dynamic accumulator to construct a group signature scheme with reasonably efficient revocation. Further improvements have been made recently by Tsudik and Xu [34].

In the past, group signature schemes aimed to satisfy a jumble of (often redundant and overlapping) security requirements: unforgeability, exculpability, traceability, coalition-resistance, no-framing, anonymity, and unlinkability [13, 8, 12, 29, 3, 9]. To untangle and simplify these messy requirements, Bellare et al. [4] investigated "minimal" security requirements for group signature schemes. This line of research is very important, as it parallels the pursuit of similar requirements for secure public key encryption schemes [26, 31, 32, 22] and secure key exchange protocols. The work in [4] showed that two security properties: full-traceability and full-anonymity are sufficient to subsume all aforementioned (seven) requirements.

In this paper, we argue that full-traceability and full-anonymity are insufficient for certain realistic group signature settings. This claim is based on the observation that group signatures are inherently applicationoriented, and, thus, cannot simply be treated as a primitive in the bare model. We identify two new requirements: leak-freedom and immediate-revocation which are necessary for a large class of commercial applications. Informally, leak-freedom means that a signer cannot 
convince anyone ${ }^{1}$ that it generated a given group signature. Whereas, immediate-revocation means that a group member's revocation results in immediate inability of generating group signatures, i.e., a revoked member cannot any further group signatures.

Why is leak-freedom important? One of the main purposes of group signatures is for an organization to conceal its internal structure. Suppose Alice is an employee of a company (say, $\mathrm{ABC}$ ) authorized to sign purchase orders and one of the suppliers is another company XYZ. If Alice - without revealing her private key or any other longterm secret - can convince XYZ that she is the signer of a given purchase order, she could obtain illegal kickbacks from XYZ as "gratitude" for her supplier selection. This sort of information leakage illustrates potential abuses of group signatures, and justifies the introduction of the leakfreedom property to block such abuses.

Why is immediate-revocation important? We continue with the previous example: any purchase order signed by Alice (on behalf of her employer ABC) for a supplier XYZ imposes certain financial and/or legal responsibilities on ABC. However, if Alice is aware of her impending lay-off, she could, in collusion with XYZ, sign fraudulent purchase orders for ABC. (Note that this problem is less grave in traditional public key infrastructures where such "poisoned" signatures cannot be attributed to anyone other than the signer herself.) This sort of abuse is possible in all current group signature schemes, unless we make very strong assumptions about underlying communication channels [23]. To this end, immediate-revocation is necessary to block such abuses without having to introduce unrealistic assumptions.

\subsection{Our Contributions}

This paper makes two noteworthy contributions. First, as mentioned above, we identify two important new security properties: leak-freedom and immediate-revocation. Second, we construct a secure and practical group signature scheme that satisfies both new and existing security properties. The proposed is very efficient: only 11 exponentiations are needed to generate a group signature, while the cost of signature verification is equivalent to verifying a single plain (i.e., non-group) signature, such as RSA. These costs are appreciably lower than those of state-of-the-art group signature schemes [9], [34].

The new scheme also offers two important advantages over previous work:

- It removes the burden of having all signers and all verifiers constantly obtaining and maintaining up-to-date

1 Except the group manager who can anyway always identify a signer. "state information" pertaining to current membership. In prior work, either all signers and all verifiers had to be aware of public revocation lists [7, 2], or all signers had to be aware of public accumulator ingredient lists [9, 34]. In the proposed scheme, the issue of dynamic group membership is fully transparent to both signers (group members) and verifiers.

- It relaxes the requirement for underlying anonymous communication channels, since a group member does not need to communicate directly with signature verifier(s). Whereas, anonymous communication channels are essential (although rarely mentioned explicitly) in previous group signature schemes.

Outline: the rest of this paper is organized as follows: the next section provides the definitions of group signatures and their desired security requirements. Section 3 presents the new group signature scheme. Section 4 discusses several extensions and Section 5 concludes the paper.

\section{Definitions}

Definition 2.1 A group signature scheme includes the following components:

Setup : a probabilistic polynomial-time algorithm that, on input of a security parameter $k$, outputs the specification of a cryptographic context including the group manager's public key $p k_{\mathcal{G M}}$ and secret key $s k_{\mathcal{G M}}$.

Join: a protocol between the group manager $\mathcal{G M}$ and a user that results in the user becoming a group member $\mathcal{U}$. Their common output includes the user's unique membership public key $p k_{\mathcal{U}}$, and perhaps some updated information that indicates the current state of the system. The user's output includes a membership secret key sk$k_{\mathcal{U}}$.

Revoke: an algorithm that, on input of a group member's identity (and perhaps her public key pk $k_{\mathcal{U}}$ ), outputs updated information that indicates the current state of the system after revoking the membership of a given group member.

Sign: a probabilistic algorithm that, on input of a group public key $p k_{\mathcal{G M}}$, a user's membership secret/public keypair $\left(s k_{\mathcal{U}}, p k_{\mathcal{U}}\right)$, and a message $m$, outputs a group signature $\delta$ of $m$.

Verify: an algorithm that, on input of a group public key $p k_{\mathcal{G M}}$, a group signature $\delta$, and a message $m$, outputs a binary value TRUE/FALSE indicating whether $\delta$ is a valid group signature (under $p k_{\mathcal{G M}}$ ) of $m$.

Open: an algorithm executed by the group manager $\mathcal{G M}$. It takes as input of a message $m$, a group signature $\delta$, the group public key $p k_{G M}$ and the group manager's secret key $s k_{\mathcal{G M}}$. It first executes VERIFY on the first three inputs and, if the $\delta$ is valid, outputs some incontestable evidence 
(e.g., a membership public key pk $k_{\mathcal{U}}$ and a proof) that allows anyone to identify the actual signer.

We now specify the desired security properties of a group signature scheme. These properties are presented informally; a more formal specification appears in the full version of this paper [21]. We note that, although we use the notions of full-traceability and full-anonymity introduced in [4], the definitions of [4] have to be amended to accommodate dynamic groups, since [4] only considers static groups.

Definition 2.2 A secure group signature scheme must have the following properties: correctness, full-traceability, full-anonymity, no-misattribution, as well as the newly introduced leak-freedom and immediate-revocation.

Correctness: any signature produced by any group member using Sign must be accepted by Verify.

Full-traceability: no subset of colluding group members (even consisting of the entire group, and even in possession of the group manager's secret key for opening signatures) can create valid signatures that cannot be opened, or signatures that cannot be traced back to some member of the colluding coalition.

Full-anonymity: it is computationally infeasible for an adversary (who is not in possession of the group manager's secret key for opening signatures) to recover the identity of the signer from a group signature, even if the adversary has access to the secret keys of all group members.

No-misattribution: it is computationally infeasible for a group manager to provably attribute a group signature to a member who is not the actual signer.

Leak-freedom: it is computationally infeasible for a signer to convince anyone that it actually signed a given message, even if it possesses all other signers' secrets, except the group manager's secret for opening signatures.

Immediate-revocation: it is computationally infeasible for a group member revoked at time t to generate a valid group signature at any time $t^{\prime}>t$.

\section{Our Construction}

Unlike most prior work, our construction is designed from a systems, rather than purely cryptographic, perspective. (Nonetheless, cryptography still plays a major role in our construction.) The main idea is the introduction of a new entity referred to as the mediation server $(\mathcal{M S}) . \mathcal{M S}$ is an on-line partially trusted server that helps in signature generation and membership revocation.

Roughly speaking, the system functions as follows: each time a group member needs to generate a signature, it somehow "identifies" itself to the mediation server which then (if the member is not revoked) produces a normal signature. This normal signature is the actual group signature and may be delivered to intended verifier(s). Nevertheless, the mere introduction of the mediation server does not imply that we can trivially obtain a group signature scheme possessing all desired security properties.

Caveat: unlike prior non-interactive group signature schemes, our scheme requires some light-weight interaction (which explains why it is able to satisfy all of the requirements). While interaction can be viewed as a drawback, we claim that it constitutes a reasonable (even small) price to pay for additional attained security properties.

\subsection{Further Motivation}

At this point, one might wonder whether a practical solution that satisfies all aforementioned requirements can be obtained in a trivial fashion. A trivial approach that satisfies all aforementioned requirements is to make the group manager an on-line entity and have it "filter" all group signature requests. Each group member has a private channel to the group manager $\mathcal{G M}$ and, for each message to be signed, it submits a message signed under its normal long-term signature key. $\mathcal{G M}$ then "translates" each incoming signature into a signature under its own well-known group signature key. The latter is then released to the verifier(s) and treated as a group signature. With this trivial solution all security properties (including leak-freedom and immediate-revocation) are satisfied and signature generation/verification costs are minimal.

However, the trivial approach requires constant ironclad security of the $\mathcal{G M}$. If this can be assured, then the trivial solution is perfect. However, having a fully trusted on-line entity is undesirable and sometimes simply unrealistic. ${ }^{2}$ A on-line $\mathcal{G M}$ would be a single point of failure in terms of both security (i.e., compromise of $\mathcal{G M}$ means compromise of the whole system) and anonymity (i.e., a dishonest $\mathcal{G M}$ can arbitrarily "open" a group signature without being held accountable). It essentially "puts all eggs in one basket." One standard way to avoid a single point of failure is to utilize distributed cryptography, which usually takes a heavy toll in system complexity, including: management, computation and communication. To avoid unnecessary complexity and subtleties, we design a system under the guidance of the well-known separation of duty principle. (See the seminal work of Clark and Wilson [20] for necessary background.) This approach, as will be shown below, facilitates a practical solution with a similar flavor of distributed security, i.e., compromise of either $\mathcal{G} \mathcal{M}$ or the newly introduced

2 For much the same reasons that Certification Authorities (CAs) are not on-line. 
mediation server $\mathcal{M S}$, but not both, does not imply complete compromise of the system.

\subsection{Model}

PARTICIPANTS: a set of group members $\mathbb{U}$ where $|\mathbb{U}|=n$, a group manager $\mathcal{G} \mathcal{M}$ who admits group members, a mediation server $\mathcal{M S}$ who revokes group members (according to $\mathcal{G} \mathcal{M}$ 's instructions) and a set of signature receivers. Each participant is modeled as a probabilistic polynomial-time interactive Turing machine. We assume that $\mathcal{M S}$ maintains a dynamic membership database DBMember-MS and $\mathcal{G M}$ maintains a similar dynamic membership database DBUserGM. Moreover, we assume that $\mathcal{M S}$ maintains a database DBSig-MS that records all signature transactions. We further assume that this database is never compromised. In practice, various uncomplicated protection mechanisms can be utilized. ${ }^{3}$

COMmunicAtion CHANNELS: all communication channels are asynchronous and under adversary's control, except the channel between $\mathcal{G} \mathcal{M}$ and $\mathcal{M S}$. In a typical system configuration, we do not assume any anonymous channels. However, we do assume that the channels between a group member $\mathcal{U} \in \mathbb{U}$ and $\mathcal{G} \mathcal{M}$, and between $\mathcal{G M}$ and $\mathcal{M S}$ are authentic. (This can be implemented via standard techniques and is thus ignored in the rest of the paper).

TRUST: precise specification of the trust model is admittedly difficult mainly because of the introduction of the new party $(\mathcal{M S})$. Nevertheless, taking into account the separation-of-duty principle, we have the following:

1. $\mathcal{G M}$ is trusted not to introduce any illegal (or phantom) group members. However, $\mathcal{G} \mathcal{M}$ may want to frame an honest group member.

2. $\mathcal{M S}$ is trusted to enforce $\mathcal{G} \mathcal{M}$ 's policy, e.g., to refuse services to any and all revoked group members; equivalently, to produce group signatures only for legitimate members. $\mathcal{M S}$ is assumed not to misbehave if such activity will be traced to it. Nonetheless, $\mathcal{M S}$ may attempt to: 1) frame an honest member into signing a message, 2) generate an unattributable group signature, and 3) compromise anonymity of an honest group member (e.g., via out-of-band means).

SECURITY DEFINITIONS: in order to accommodate the new entity $(\mathcal{M S})$, we need to slightly amend some parts of Definition 2.2 for our setting. The changes are minimal

3 For example, $\mathcal{M S}$ can use a decoy technique and insert $(n-1)$ well-formed dummy transaction records for each genuine one. Alternatively, the database can be kept encrypted with the encryption key protected using some advanced cryptographic techniques. These and other approaches and their respective merits are discussed in the full version of this paper [21]. but necessary for the sake of clarity. (The rest of the definitions remains unchanged.)

Definition 3.1 Full-traceability: the set of colluders can additionally include $\mathcal{M S}$.

Full-anonymity: the adversary is additionally allowed to have access to the secret key $(s)$ of $\mathcal{M S}$.

Leak-freedom: it is infeasible for a signer to convince anyone (except $\mathcal{M S}$ ) that it generated a group signature; it is infeasible for $\mathcal{M S}$ to convince anyone (except $\mathcal{G} \mathcal{M})$ that a certain group member generated a group signature.

\subsection{Accountable Designated Verifier Signatures}

We introduce the notion of accountable designated verifier signatures (ADVS) that will serve as the building block in our group signature scheme. This notion is an enhancement of the private contract signatures introduced in [25]. Informally, a private contract signature is a designated verifier signature that can be converted into a universallyverifiable signature by either the signer or a trusted third party appointed by the signer. An accountable designated verifier signature scheme, on the other hand, emphasizes the trusted third party's capability to identify the actual signer of a valid signature.

Definition 3.2 Let $P_{i}$ and $P_{j}$ be two distinct entities and $T$ be a trusted third party. Suppose $P_{i}$ is the signer and $P_{j}$ is the verifier. An accountable designated verifier signature scheme, ADVS, is a tuple of polynomial-time algorithms (ADVS-Sign, ADVS-Ver, ADVS-Proof) defined as follows:

ADVS-Sign: an algorithm executed by $P_{i}$ on message $m$ to output an ADVS: $\delta=\mathrm{ADVS}-\operatorname{Sign}_{P_{i}}\left(m, P_{j}, T\right)$.

ADVS-Ver: an algorithm which allows $P_{j}$ to verify the validity of an input tag $\delta$ on message $m$, such that:

ADVS-Ver $\left(m, P_{i}, P_{j}, T ; \delta\right)=$

$$
\left\{\begin{array}{l}
\text { TRUE if } \delta=\text { ADVS-Sign } P_{i}\left(m, P_{j}, T\right) \\
\text { FALSE otherwise }
\end{array}\right.
$$

ADVS-Proof : an algorithm executed by $T$ on input $P_{i}$, $m, P_{j}$, and tag $\delta$. It outputs a proof for the predicate SignedBy $\left(\delta, P_{i}\right)$ which is TRUE if $\delta$ is produced by $P_{i}$, and FALSE otherwise.

We require that, if $\delta=\mathrm{ADVS}-\operatorname{Sign}_{P_{i}}\left(m, P_{j}, T\right)$, then: ADVS-Ver $\left(m, P_{i}, P_{j}, T ; \delta\right)=$ TRUE and $T$ always outputs a proof for $\operatorname{SignedBy}\left(\delta, P_{i}\right)$ being TRUE.

Definition 3.3 An ADVS scheme is secure if the following properties are satisfied:

Unforgeability of ADVS-Sign $P_{i}\left(m, P_{j}, T\right)$ : for any $m$, it is infeasible for any $P \notin\left\{P_{i}, P_{j}\right\}$ to produce $\delta$, such that ADVS-Ver $\left(m, P_{i}, P_{j}, T ; \delta\right)=$ TRUE. 
Non-transferability of ADVS-Sign $P_{i}\left(m, P_{j}, T\right):$ for $P_{j}$, there is a polynomial-time forgery algorithm which, for any $m, P_{i}$, and $T$, outputs $\delta$ such that ADVS-Ver $\left(m, P_{i}, P_{j}, T ; \delta\right)=$ TRUE.

Unforgeability of the proof for $\operatorname{SignedBy}\left(\delta, P_{i}\right)$ : for any $\delta=\operatorname{ADVS}-\operatorname{Sign}_{P_{i}}\left(m, P_{j}, T\right)$, it is infeasible for any $P \notin\left\{T, P_{i}\right\}$ to produce a proof for $\operatorname{SignedBy}\left(\delta, P_{i}\right)$ being TRUE.

We need to further differentiate between the case that $P_{i}$ is able to produce a proof for $\operatorname{SignedBy}\left(\delta, P_{i}\right)$ and the case that $P_{i}$ is unable to do so. Although the former is desirable in the context of private contract signatures, it is undesirable in our setting. This is because private contract signatures only intend to prevent $P_{j}$ transferring the one bit information $\operatorname{SignedBy}\left(\delta, P_{i}\right)$ by any means. Whereas, it would be very useful in our context for both $P_{i}$ and $P_{j}$ not to be able to leak the bit: $\operatorname{SignedBy}\left(\delta, P_{i}\right)$. Thus, we have the following definition:

Definition 3.4 An ADVS scheme is strongly-secure if, in addition to being a secure ADVS scheme, it ensures that a signer $P_{i}$ cannot produce a proof for $\operatorname{SignedBy}\left(\delta, P_{i}\right)$ with non-negligible probability, where $\delta=$ ADVS-Sign $P_{i}\left(m, P_{j}, T\right)$.

A strongly-secure ADVS scheme can allow us to easily construct a simple leak-free group signature system. Unfortunately, we do not know how to construct such a scheme, so we leave it as an interesting open problem. In order to facilitate a group signature scheme that is leakfree with immediate-revocation, we utilize a secure ADVS scheme that is based on the ideas in [25]. Due to space limitation, the details appear in the full version of this paper [21].

\subsection{Leak-free Group Signatures with Immediate Revocation}

We are finally ready to present a concrete construction. The basic operation of our scheme is as follows. Given a message $m$, a group member $\mathcal{U}_{i}$ presents an ADVS $\delta=$ ADVS-Sign $\mathcal{U}_{i}(m, \mathcal{M S}, \mathcal{G M})$ to $\mathcal{M S}$ requesting (and obtaining) a plain signature $\sigma=\operatorname{Sign}_{\mathcal{M S}}(m)$. The latter is viewed as a group signature, for which there is a single group-wide verification key. Note that, since $\mathcal{G} \mathcal{M}$ plays the role of a trusted third party in the ADVS scheme, it can hold an actual signer accountable. We also note that our trust model implies that there are no issues with the fair exchange of $\delta=$ ADVS-Sign $\mathcal{U}_{i}(m, \mathcal{M S}, \mathcal{G M})$ for $\sigma=\operatorname{Sign}_{\mathcal{M S}}(m)$, even if $\sigma$ is returned to the user. Following the definition of group signatures, our mediated group signature scheme consists of the following procedures:
SETUP : this procedure initializes a group manager $\mathcal{G} \mathcal{M}$ and a mediation server $\mathcal{M S}$.

1. $\mathcal{G} \mathcal{M}$ chooses a system wide security parameter $\kappa$. Based on it, $\mathcal{G M}$ chooses a discrete-logarithm based crypto-context as in a normal Schnorr signature setting. The parameter $\kappa$ and the crypto-context are followed system-wide by all group members, the $\mathcal{M S}$ and $\mathcal{G M}$ itself. $\mathcal{G M}$ initializes a set $\mathbb{U}$ wherein each group member will be assigned a unique identity $\mathcal{U}_{i} \in \mathbb{U}$. $\mathcal{G} \mathcal{M}$ then instantiates an ADVS scheme ADVS as well as its own public/private key-pair: $\left\langle Y_{\mathcal{G M}}=g^{X_{\mathcal{G M}}}, X_{\mathcal{G M}}\right\rangle$. Finally, $\mathcal{G} \mathcal{M}$ initializes the database DBUser-GM.

2. The initialization of the mediation server $\mathcal{M S}$ consists of the following: choose a pair of ADVS public and private keys $\left\langle Y_{\mathcal{M S}}=g^{X_{\mathcal{M S}}}, X_{\mathcal{M S}}\right\rangle$ in the same crypto-context as in step 1. Choose a pair of keys for a normal digital signature scheme $\mathcal{S I G}=\left(\right.$ Gen, $\left.\operatorname{Sign}_{\mathcal{M S}}, \operatorname{Ver}_{\mathcal{M S}}\right)$ that is secure against adaptive chosen-message attack [27]. Denote by $\left\langle p k_{\mathcal{M S}}, s k_{\mathcal{M S}}\right\rangle$ the pair of group signature verification and generation keys, where $p k_{\mathcal{M S}}$ is publicly known. ${ }^{4}$ Initialize databases DBMember-MS and DBSig-MS.

JOIN : whenever $\mathcal{G} \mathcal{M}$ decides to admit a new member, it first assigns a unique identity $\mathcal{U}_{i} . \mathcal{U}_{i}$ generates its ADVS public/private key-pair: $\left\langle Y_{\mathcal{U}_{i}}=g^{X \mathcal{U}_{i}}, X_{\mathcal{U}_{i}}\right\rangle . Y_{\mathcal{U}_{i}}$ is then registered at the $\mathcal{G M}$ and $\mathcal{M S}$; both DBMember-GM and DBMember-MS are updated accordingly.

SIGN : whenever a group member $\mathcal{U}_{i}$ wants to generate a group signature on message $m$, the following protocol is executed:

1. $\mathcal{U}_{i}$ sends $\mathcal{M S}$ an ADVS $\delta=\operatorname{ADVS}-\operatorname{Sign}_{\mathcal{U}_{i}}(m, \mathcal{M S}, \mathcal{G M})$ over an insecure public (and unauthenticated) channel.

2. Upon receipt, $\mathcal{M S}$ retrieves $\mathcal{U}_{i}$ 's public key $Y_{\mathcal{U}_{i}}$ from its database DBMember-MS. If no entry is found, $\mathcal{M S}$ simply ignores the request; otherwise, $\mathcal{M S}$ verifies $\delta$ by checking whether ADVS-Ver $(m, \mathcal{M S}, \mathcal{G M} ; \delta)=$ TRUE. If it is, $\mathcal{M S}$ then produces a normal signature $\gamma=\operatorname{Sign}_{\mathcal{M S}}(m)$ and inserts a new record $\left(\mathcal{U}_{i}, \delta, \gamma\right)$ into its database DBSig-MS. The signature $\gamma$ is a group signature on message $m$.

VERIFY : given $p k_{\mathcal{M S}}$, the public group signature verification key of $\mathcal{M S}$, and a tag $\gamma$, anyone can establish whether $\gamma$ is a valid (group) signature by running $\operatorname{Ver}_{\mathcal{M S}}$ on input: $p k_{\mathcal{M S}}, m$, and $\gamma$.

4 We assume that $\mathcal{M S}$ knows $s k_{\mathcal{M S}}$ in its entirety so as to prevent attacks due to incorrect system initialization. This can be ensured by utilizing techniques due to [35]. 
REVOKE : whenever $\mathcal{G} \mathcal{M}$ decides to revoke membership of $\mathcal{U}_{i}$, it first updates DBUser-GM indicating that $\mathcal{U}_{i}$ is revoked and $\log$ s the necessary information. $\mathcal{G M}$ then informs $\mathcal{M S}$ that $\mathcal{U}_{i}$ is revoked. and $\mathcal{M S}$ proceeds to delete the entry $\left(\mathcal{U}_{i}, Y_{\mathcal{U}_{i}}\right)$ from its database DBMember-MS. Consequently, all subsequent signature requests from $\mathcal{U}_{i}$ will be rejected by $\mathcal{M S}$.

OPEN : whenever $\mathcal{G} \mathcal{M}$ decides to identify the actual signer of signature $\gamma$ on message $m$, the following protocol is executed by $\mathcal{G M}$ and $\mathcal{M S}$ :

1. $\mathcal{G M}$ sends $\gamma$ to $\mathcal{M S}$ via an authenticated channel.

2. $\mathcal{M S}$ retrieves $\left(\mathcal{U}_{i}, \delta, \gamma\right)$ from its database and sends it to $\mathcal{G} \mathcal{M}$ via the same authenticated channel.

3. $\mathcal{G M}$ checks whether ADVS-Ver $(m, \mathcal{M S}, \mathcal{G M} ; \delta)=$ TRUE. If so, $\mathcal{G M}$ executes ADVS-Proof to produce a proof for $\operatorname{SignedBy}\left(\delta, \mathcal{U}_{i}\right)$; otherwise, $\mathcal{G} \mathcal{M}$ concludes that $\mathcal{M S}$ is cheating.

Remark: the above protocol does not specify how a group signature is sent to its intended verifier(s). There are two options. One way that allows us to completely get rid of the anonymous channels is to let $\mathcal{M S}$ send $\gamma$ directly to the verifier(s). In this case, there may be a need for a random delay to address potential traffic analysis (see further discussion below). Note that this kind of delay already exists in the anonymous channels currently available. The other way is to let $\mathcal{M S}$ broadcast $\gamma$ so that $\mathcal{U}_{i}$ can receive and re-send $\gamma$ to the verifier(s) via an anonymous channel. We prefer the former, however, a detailed analysis is deferred to [21].

\subsection{Security Analysis}

Theorem 3.1 Our scheme satisfies the requirements specified in Definition 3.1, namely correctness, fulltraceability, full-anonymity, no-misattribution, leakfreedom, and immediate-revocation.

A formal proof of this theorem appears in a full version of this paper [21]. Intuitively, the theorem holds because the final group signatures are generated using a standard signature algorithm, and ADVS makes it infeasible for the group members or $\mathcal{M S}$ to cryptographically prove the linkage between a signature request and the resulting group signature.

\section{Extension and Discussion}

Enhancing anonymity against traffic analysis. Our scheme does not assume that the channel between a group member $\mathcal{U}$ and the mediation server $\mathcal{M S}$ is authenticated or anonymous. This is because $\mathcal{M S}$ may have incentives to cheat an outsider, which, in turn, implies:
1. Even if the adversary eavesdrops on all channels, there could still be an out-of-band channel between a group member and $\mathcal{M S}$. Thus, the adversary could still be fooled.

2. $\mathcal{M S}$ can easily cheat an outsider by injecting a fake ADVS into the network or its database DBSig-MS.

However, an adversary might know that $\mathcal{M S}$, while not being trusted to preserve anonymity, does not always inject fake traffic into the network. Then, an adversary has a good chance of compromising anonymity of some honest group members by means of traffic analysis. Fortunately, this can be avoided by using standard techniques, such as end-to-end encryption and traffic padding.

On strongly-secure ADVS vs. secure ADVS. In our construction we utilized an ADVS that is secure, but not strongly-secure. Consequently, we assume the secrecy of $\mathcal{M S}$ 's storage, particularly DBSig-MS consisting of all signature transaction entries. This is necessary in order to avoid the following attack: If an attacker has access to an entry in DBSig-MS, then Alice can convince the attacker that she generated a group signature $\operatorname{Sign}_{\mathcal{M S}}(m)$. Clearly, if a strongly-secure ADVS is utilized, we can achieve strictly stronger security that Alice is unable to convince $\mathrm{XYZ}$ that she generated a signature, even if XYZ has access to the corresponding entry in DBSig-MS. It seems non-trivial to ensure secrecy of DBSig-MS, while preserving other desirable properties. We continue investigating technical mechanisms that can replace this rather strong assumption regarding database secrecy [21].

Denial-of-Service (DoS) attacks. Recall than $\mathcal{M S}$ always performs a number of modular exponentiations before it is able to determine whether an incoming ADVS is valid. This opens the door for DoS attacks on $\mathcal{M S}$. To counter such attacks, we suggest a simple solution. The idea is to let each $\mathcal{U}_{i}$ and $\mathcal{M S}$ share a unique secret key $w_{i}$. Each signature request from $\mathcal{U}_{i}$ must also be accompanied by an authentication token computed over the request with the key $w_{i}$. When processing a request, $\mathcal{M S}$ verifies the authentication token before performing much more expensive validation of the ADVS. Note that this additional authentication is does not in any way influence security properties of our scheme. Of course, this is only a partial solution since an adversary can still mount a DoS attack on the $\mathcal{M S}$ 's network interface.

\subsection{Related Work}

This paper can be viewed as one of many efforts pursuing practical and secure group signature or identity escrow schemes $[18,19,13,29,1,11]$, as well as anonymous credential systems $[16,17,30,10,14]$. Among them, the prior work most relevant to this paper is [11], which presented an 
identity escrow scheme (and a corresponding group signature scheme) with the appointed verifier property. The motivation was to obtain a scheme where a group member can only convince one or more appointed verifiers of its membership, while no other party can verify membership even if the signer cooperates fully.

Note that there is a difference between the appointed verifier property in [11] and the leak-freedom property specified in this paper. Specifically, the scheme in [11], by definition, allows a signer to convince the designated verifiers that it is authorized to conduct the relevant transactions. Cast this in our example scenario, Alice can always convince XYZ that she is authorized to sign purchase orders. This capability can result in the leakage (outlined in Section 1) we meant to avoid! Besides achieving the strictly stronger leak-freedom, our scheme is more efficient than [11] which requires both a signer and a verifier to compute more than $17 k$ exponentiations, where $k$ is a security parameter (say, $k=80$ ). Moreover, membership revocation is not supported in [11], whereas, we achieve immediaterevocation which has only been explored in the context of traditional PKI-s [6].

A credential system is a system where users can obtain credentials from organizations and demonstrate possession of these credentials $[16,17,30,10,14]$. Chaum and Evertse [17] presented a general scheme using a semi-trusted TTP common to multiple organizations. However, their scheme is impractical. The credential system by Lysianskaya et al. [30] captures many of the desirable properties. Camenisch and Lysianskaya [10] presented a better solution with ingredients from a secure group signature scheme of [1]. The prototype implementation of [10] was done by Camenisch and van Herreweghen [14]. This scheme requires both signers and verifiers to compute 22 modular exponentiations. Their advanced scheme which provides all-or-nothing nontransferability (to discourage a signer from sharing its credentials with other parties) requires both signer and verifier to compute 200 exponentiations.

The notion of abuse-freedom or abuse-freeness [25] is weaker than leak-freedom because the former intends only to prevent the designated verifier from being able to transfer the information about the actual signer, whereas the latter intends to prevent a signer as well as the designated verifier from being able to transfer the same information. Finally, we remark that leak-freedom is similar to the property called receipt-freedom or receipt-freeness in the context of voting schemes [5, 28], and its closely related variants called deniable encryption [15] and deniable proof [24].

\section{Conclusion}

We identified two properties, namely leak-freedom and immediate-revocation, that are crucial for a large class of group signature applications. We also constructed a scheme that achieves all of traditional and newly-introduced goals by following a systems architectural approach. Our scheme is practical and easy to implement, because it needs only 11 exponentiations for a group member to generate a group signature and one normal signature verification for its validation. Another contribution of this paper is the relaxation on the requirement for anonymous communication channels, which are essential in all previous schemes.

In addition to the already mentioned issues (including the protection of the server databases, and the robustness against denial-of-service attacks) that need further investigations, our work also incurs some problems that are interesting in an even more general context:

1. How to construct a practical strongly-secure ADVS scheme?

2. How to construct a leak-free group signature scheme with immediate revocation that does not rely on a mediation server? Although we believe that the existence of a mediation server is more realistic than the existence of (for instance) a time-stamping service, it is nevertheless conceivable that other alternatively constructions could fit well into different specific application scenarios.

3. How to achieve a stateless $\mathcal{M S}$ ? This is not trivial because, otherwise, the binding of an ADVS signature (or any other token with the desired properties) and a normal signature would allow $\mathcal{M S}$ to convince an outsider of the identity of the actual signer.

\section{References}

[1] G. Ateniese, J. Camenisch, M. Joye, and G. Tsudik. A practical and provably secure coalition-resistant group signature scheme. In CRYPTO'2000, pages 255-270.

[2] G. Ateniese, D. Song, and G. Tsudik. Quasi-efficient revocation of group signatures. In Financial Crypto'02).

[3] G. Ateniese and G. Tsudik. Some open issues and new directions in group signatures. In Financial Crypto'99).

[4] M. Bellare, D. Micciancio, and B. Warinschi. Foundations of group signatures: Formal definitions, simplified requirements, and a construction based on general assumptions. In EuroCrypto'2003.

[5] J. Benaloh and D. Tuinstra. Receipt-free secret-ballot election (extended abstract). In STOC, 1994.

[6] D. Boneh, X. Ding, G. Tsudik, and C. Wong. A method for fast revocation of public key certificates and security capabilities. In 10th USENIX Security Symposium, 2001.

[7] E. Bresson and J. Stern. Group signatures with efficient revocation. In International Workshop on Practice and Theory in Public Key Cryptography (PKC) '2001.

[8] J. Camenisch. Group Signature Schemes and Payment Systems Based on the Discrete Logarithm Problem. PhD thesis, ETH Zurich, 1998. 